ural and supplemented recovery of human microbial communities. Nat Rev Microbiol. 2011;9(1):27-38.

4. Metchnikoff E. The Nature of Man: Studies in Optimistic Philosophy. New York, New York, USA: GP Putman's Sons; 1908.

5. Agheyisi R. The Probiotics Market: Ingredients, Supplements, Foods. BCC Research. Report \#FOD035B. http://www.bccresearch.com/report/ probiotic-supplements-food-fod035b.html. Updated June 2008. Accessed April 8, 2011.

6. Aureli P, et al. Probiotics and health: an evidencebased review [published online ahead of print February 22, 2011]. Pharmacol Res. doi:10.1016/ j.phrs.2011.02.006.

7. Yan F, Polk DB. Probiotics: progress toward novel therapies for intestinal diseases. Curr Opin Gastroenterol. 2010;26(2):95-101.

8. Corr SC, Li Y, Riedel CU, O’Toole PW, Hill C, Gahan CG. Bacteriocin production as a mechanism for the antiinfective activity of Lactobacillus salivarius UCC118. Proc Natl Acad Sci U S A. 2007;
104(18):7617-7621.

9. Lebeer S, Vanderleyden J, De Keersmaecker SC. Host interactions of probiotic bacterial surface molecules: comparison with commensals and pathogens. Nat Rev Microbiol. 2010;8(3):171-184.

10. O'Mahony C, et al. Commensal-induced regulatory $\mathrm{T}$ cells mediate protection against pathogenstimulated NF-kappaB activation. PLoS Pathog. 2008;4(8):e1000112.

11. Goldin BR, Gorbach SL. Clinical indications for probiotics: an overview. Clin Infect Dis. 2008; 46(suppl 2):S96-S100.

12. Preidis GA, Hill C, Guerrant RL, Ramakrishna BS, Tannock GW, Versalovic J. Probiotics, enteric and diarrheal diseases, and global health. Gastroenterology. 2011;140(1):8-14

13. Kruis W, et al. Maintaining remission of ulcerative colitis with the probiotic Escherichia coli Nissle 1917 is as effective as with standard mesalazine. Gut. 2004;53(11):1617-1623.

14. Tursi A, Brandimarte G, Giorgetti GM, Forti G,
Modeo ME, Gigliobianco A. Low-dose balsalazide plus a high-potency probiotic preparation is more effective than balsalazide alone or mesalazine in the treatment of acute mild-to-moderate ulcerative colitis. Med Sci Monit. 2004;10(11):PI126-PI131.

15. Yan F, Polk DB. Probiotic bacterium prevents cytokine-induced apoptosis in intestinal epithelial cells. J Biol Chem. 2002;277(52):50959-50965.

16. Yan F, Cao H, Cover TL, Whitehead R, Washington MK, Polk DB. Soluble proteins produced by probiotic bacteria regulate intestinal epithelial cell survival and growth. Gastroenterology. 2007;132(2):562-575.

17. Seth A, Yan F, Polk DB, Rao RK. Probiotics ameliorate the hydrogen peroxide-induced epithelial barrier disruption by a PKC- and MAP kinase-dependent mechanism. Am J Physiol Gastrointest Liver Physiol. 2008;294(4):G1060-G1069.

18. Yan F, et al. Colon-specific delivery of a probioticderived soluble protein ameliorates intestinal inflammation in mice through an EGFR-dependent mechanism. J Clin Invest. 2011;121(6):2242-2253.

\title{
Neuroanatomy of body weight control: lessons learned from leptin
}

\author{
Diana L. Williams ${ }^{1}$ and Michael W. Schwartz ${ }^{2}$
}

${ }^{1}$ Department of Psychology and Program in Neuroscience, Florida State University, Tallahassee, Florida, USA. ${ }^{2}$ Diabetes and Obesity Center of Excellence, Department of Medicine, University of Washington, Seattle, Washington, USA.

\begin{abstract}
Rather than arising from the passive accumulation of excess calories, obesity is a state in which the biologically defended level of body fat stores increases due to defects in the homeostatic process that matches food intake and energy expenditure over time. By deleting leptin receptors from distinct brain regions and neuronal subsets, researchers are beginning to identify the neuroanatomical substrates responsible for this regulation. In this issue of the JCI, Scott et al. demonstrate that loss of leptin receptors in a subset of hindbrain neurons increases food intake in mice, but, unlike what is observed when leptin receptors are deleted from hypothalamic neurons, these mice compensate by increasing energy expenditure and hence do not become obese. Although many brain areas can regulate energy intake and/or energy expenditure, it is likely that only a small subset of neurons actively matches the two over time. It is vital to clarify how this works if we are to improve our understanding of obesity pathogenesis and options available for its treatment.
\end{abstract}

The alarmingly high prevalence of obesity and related metabolic disorders has emerged as one of the most costly public health problems facing developed countries. The lack of effective treatment options exacerbates this problem and reminds us that despite steady progress in our understanding of neural and endocrine pathways controlling energy balance, our insight into mechanisms that underlie common forms of obesity remains quite limited.

Conflict of interest: Michael W. Schwartz received income as an advisor to Pfizer Inc.

Citation for this article: J Clin Invest. 2011;

121(6):2152-2155. doi:10.1172/JCI58027.
Leptin, a hormone secreted by adipocytes in proportion to fat mass, plays a critical role in energy homeostasis by acting through its neuronal receptors in multiple brain areas to decrease food intake and increase energy expenditure (1). Because mutations that disrupt either leptin production or leptin receptors cause extreme hyperphagia and obesity in rodents and humans, there is little question of its physiological importance. However, the question of how leptin's many effects are mediated remains unresolved. One approach to addressing this question involves deletion of the gene encoding the leptin receptor in specific cell types using mouse molecular genetics. In this issue, Scott and colleagues describe mice in which leptin receptor expression was deleted exclusively in hindbrain neurons that express the transcription factor paired-like homeobox $2 b$ (Phox2b) (2). As predicted, these mice are hyperphagic, but, unlike the obese phenotype of mouse models in which leptin receptors were deleted from hypothalamic neuronal populations (3-5), body weight is not substantially altered because the increased caloric intake is offset by increased energy expenditure. This outcome points to meaningful differences in the roles of hypothalamus and hindbrain as targets of leptin action.

\section{Brain mechanisms that control feeding behavior}

Neural control of food intake involves the integration of diverse signals: long-term signals related to stored fuel, including leptin; short-term signals that arise from the gastrointestinal tract in response to ingested nutrients; and reward-related factors, such as the hedonic and incentive value of the food (6). The brain uses these signals to make decisions about eating on a meal-to-meal basis in ways that serve the longer-term goal of maintaining body weight within a stable range. Since the mid-20th century, the hypothalamus has 


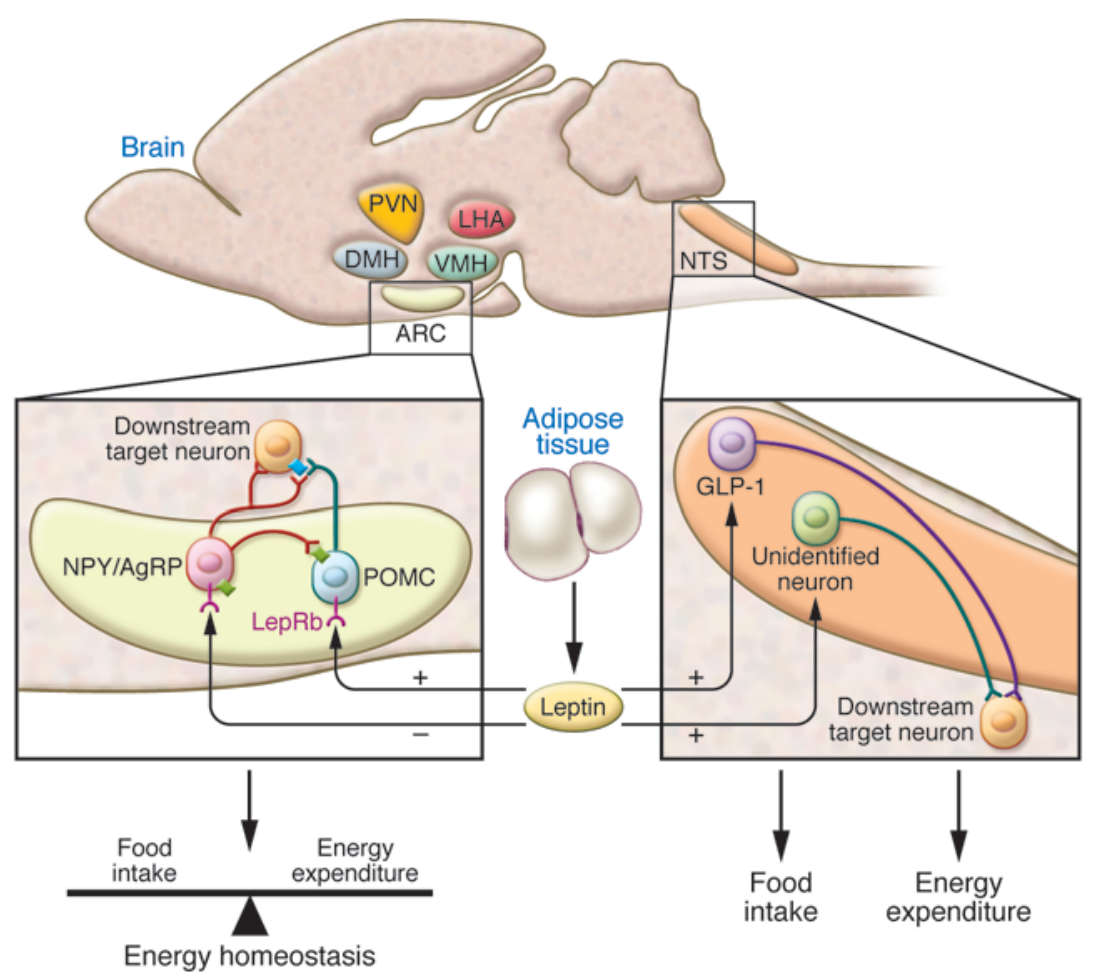

been considered a key player in long-term body weight regulation, a view reinforced by the discovery that leptin receptor expression in ventromedial hypothalamic areas is especially high (1). Short-term control of meal size, on the other hand, is widely considered to reside primarily in the caudal brainstem (7). Thus, feeling "full" after a meal arises not from leptin action but from secretion of gastrointestinal peptides, such as cholecystokinin (CCK), that promote meal termination by activating neurons in the hindbrain nucleus of the solitary tract (NTS) and area postrema (AP) (7).

Insight into the role of the caudal brainstem in food intake control derives in part from the chronically maintained decerebrate rat model in which the brain is transected at the level of the superior colliculus, eliminating neural communication between forebrain and hindbrain. Such studies reveal that treatments influencing meal size in the short term, including gastrointestinal nutrient infusion and intraperitoneal injection of CCK, suppress food intake comparably in decerebrate rats and controls (8). Yet this is not the case when the response to a longer-term metabolic challenge is assessed. For example, 24 hours of food deprivation causes intact rats to ingest more food than usual at the first opportunity to eat, but decerebrated rats fail to show this refeeding hyperphagia (8). Thus, the isolated caudal brainstem is sufficient for meal size control by shortterm signals, but input from the forebrain is required for the behavioral response to a decrease in the availability of stored fuel.

\section{Leptin action in hypothalamus and hindbrain}

Although research on leptin action has emphasized the role of hypothalamic mediators, leptin receptors are expressed throughout the brain, and extrahypothalamic receptor populations can clearly mediate leptin effects. Activation of leptin receptors in the hindbrain reduces food intake while increasing energy expenditure such that body weight is reduced (9). Moreover, the mechanism whereby leptin action in both hypothalamus and hindbrain reduces overall food intake involves an enhanced response to the satiating effect of short-term signals such as CCK (10-13).

In the hypothalamus, leptin action depends critically (though not exclusively) on two arcuate nucleus (ARC) neuronal types: those expressing proopiomelanocortin (POMC), the precursor for the anorexigenic neuropeptide $\alpha$-melanocyte-stimulating hormone, and those coexpressing agouti-related protein (AgRP) and neuropeptide Y (NPY), both orexigenic neuro-

\section{Figure 1}

Hypothalamic and hindbrain neurocircuits that regulate food intake and energy expenditure in response to input from the adipocyte hormone leptin. Although leptin reduces food intake and body weight through actions at both sites, neurons in the ARC appear to actively integrate input from both sides of the energy balance equation, whereas the control over food intake and energy expenditure by NTS neurons may be distinct and separable. $\mathrm{DMH}$, dorsomedial hypothalamic nucleus; LHA, lateral hypothalamic area; LepRb, long-form leptin receptor; PVN, paraventricular nucleus; $\mathrm{VMH}$, ventromedial hypothalamic nucleus. peptides (1) (Figure 1). Far less is known about neuronal populations that mediate leptin's effects in the hindbrain. POMC is expressed in the NTS, but the sensitivity of hindbrain POMC neurons to leptin is controversial $(14,15)$. AgRP is not expressed in hindbrain, and leptin receptors are not expressed by hindbrain NPY neurons (16). In mouse (but not in rat) hindbrain, leptin receptors are expressed in glucagon-like 1 peptide (GLP-1) neurons, and leptin regulates expression of mRNA for proglucagon, the peptide precursor of GLP-1 (17, 18). Thus, despite similarities in feeding effects of forebrain and hindbrain leptin treatment, the neural circuitry engaged by leptin differs across these regions.

High-fat diet (HFD) feeding offers another example of a difference between leptin action in hypothalamus versus the hindbrain. During HFD feeding, body fat mass and circulating leptin levels increase, and reduced sensitivity to exogenous leptin - leptin resistance - can develop rapidly. To the extent that leptin resistance contributes to obesity pathogenesis in this setting, as many have suggested (19), the deficit appears localized to the hypothalamus, because HFD-induced impairment of leptin signaling occurs in the ARC but not the NTS (20).

Scott and colleagues' (2) deletion of leptin receptors from Phox $2 \mathrm{~b}$-expressing 
neurons is the first report of cell type-specific manipulation of leptin signaling in mouse hindbrain. Although the neurochemical identity of the hindbrain cells targeted remains to be fully ascertained, GLP-1 neurons are among those in which leptin receptor deletion occurred. The finding that Phox $2 b$ Cre Leprflox/flox (PC flox) mice display both increased food intake and an exaggerated hyperphagic response to an overnight fast suggests that in normal mice, leptin signaling in GLP-1 and/or other hindbrain neurons constrains these behaviors. Yet the body fat content of PC flox mice was not increased, evidently because their metabolic rate increased so as to maintain neutral energy balance. Thus, although leptin action in Phox2b-expressing hindbrain neurons appears to play a physiological role to limit food intake, loss of leptin signaling in these cells does not prevent the detection of and compensation for this perturbation of energy balance.

The conclusion that increased energy expenditure in PC flox mice results from, rather than causes, hyperphagia derives from the finding that their hypermetabolic phenotype was eliminated during a fast and hence depends upon food consumption. Interestingly, the ability of leptin to reduce food intake and body weight was not attenuated in these mice, suggesting that leptin signaling in the subset of hindbrain neurons that express the Phox $2 \mathrm{~b}$ promoter is not required for leptin's anorexic effect. Moreover, the differences of food intake and energy expenditure between controls and PC flox mice disappeared when they were placed on a HFD.

This constellation of features is unique, and it differs in important ways from the phenotypes of mice in which leptin receptors are deleted from hypothalamic neurons. Mice lacking leptin receptors in POMC neurons eat the same amount of food as controls but have increased body weight as a result of reduced energy expenditure (5). Leptin receptor deletion in neurons of the ventromedial hypothalamic nucleus produces a similar phenotype, although energy intake increases when the mice are placed on a HFD (4). Deletion of leptin receptors in NPY/AgRP neurons also increases body weight via yet another a mechanism involving reductions of locomotor activity and body temperature, and a somewhat greater obesity results when leptin receptors are deleted from both NPY/AgRP and POMC cells, due to combined effects of hyperpha- gia and reduced energy expenditure (3). Therefore, loss of hypothalamic leptin signaling consistently favors positive energy balance and obesity, whereas PC flox mice maintain near-normal energy balance because increases of energy intake and expenditure offset one another.

It is worth noting that less selective leptin receptor deletion strategies invariably have more robust effects. For example, targeted deletion of leptin receptors from all hypothalamic neurons causes pronounced hyperphagia and obesity (21), and Hayes and colleagues (10) recently showed that shRNAi-induced knockdown of leptin receptor expression in the NTS and AP increases food intake and body weight, although the effect is modest by comparison. The differences between the phenotypes of these and aforementioned, more selective models highlights the complexity of the circuitry that mediates leptin's effects and emphasizes both the importance of and the limitations inherent in cell type-specific strategies for delineating the roles of distinct neuronal populations.

\section{The importance of coupling energy intake and expenditure}

The stability with which body fat stores are maintained in normal animals in the face of continuously changing energy demands testifies to the remarkable precision of systems that govern energy balance. This fact reminds us that obesity arises from disordered energy homeostasis - an upward resetting of the defended level of body weight - rather than from passive accumulation of excess calories. That food intake and energy expenditure can be uncoupled when leptin receptors are deleted from some neural circuits but not from others highlights distinct roles played by different brain areas in the control of energy balance. This concept is consistent with a large number of studies, in which obesity is induced by interventions directed at specific brain areas. Based on the evidence presented by Scott et al. (2) and others discussed here, we speculate that although many brain areas can regulate food intake and energy expenditure, the dynamic matching of these two variables in the service of energy homeostasis is a function that may be unique to hypothalamic neurons. Testing this hypothesis will help to identify mechanisms by which the brain compensates for changes of food intake or energy expenditure. Because dietinduced weight loss itself triggers compen- satory metabolic and behavioral responses that promote the regain of that weight, the issue is of fundamental clinical relevance. Hoped-for breakthroughs in obesity treatment may remain elusive until we gain a clearer understanding of how this compensation occurs.

\section{Acknowledgments}

This work was supported by National Institute of Diabetes and Digestive and Kidney Diseases grants DK068384, DK083042, and DK052989 (to M.W. Schwartz); Nutrition Obesity Research Center (DK035816); Diabetes Endocrine Research Center (P30 DK17047); Mouse Metabolic Phenotyping Center (U24 DK076126) at the University of Washington; and National Institute of Digestive and Kidney Diseases grant DK078779 (to D.L. Williams).

Address correspondence to: Michael W. Schwartz, Diabetes and Obesity Center of Excellence, University of Washington Medicine at South Lake Union, 815 Mercer Street, Box 358055, Seattle, Washington 98109, USA. Phone: 206.897.5288; Fax: 206.341.5293; E-mail: mschwart@ u.washington.edu.

1. Porte D Jr, Baskin DG, Schwartz MW. Leptin and insulin action in the central nervous system. Nutr Rev. 2002;60(10 pt 2):S20-S29.

2. Scott MM, Williams KW, Rossi J, Lee CE, Elmquist JK. Leptin receptor expression in hindbrain Glp-1 neurons regulates food intake and energy balance in mice. J Clin Invest. 2011;121(6):2413-2421.

3 . van de Wall E, et al. Collective and individual functions of leptin receptor modulated neurons controlling metabolism and ingestion. Endocrinology. 2008;149(4):1773-1785

4. Dhillon $\mathrm{H}$, et al. Leptin directly activates SF1 neurons in the $\mathrm{VMH}$, and this action by leptin is required for normal body-weight homeostasis. Neuron. 2006;49(2):191-203.

5. Balthasar $\mathrm{N}$, et al. Leptin receptor signaling in POMC neurons is required for normal body weight homeostasis. Neuron. 2004;42(6):983-991.

6. Zheng H, Berthoud HR. Neural systems controlling the drive to eat: mind versus metabolism. Physiology (Bethesda). 2008;23:75-83.

7. Woods SC. Gastrointestinal satiety signals I. An overview of gastrointestinal signals that influence food intake. Am J Physiol Gastrointest Liver Physiol. 2004;286(1):G7-G13.

8. Grill HJ, Kaplan JM. The neuroanatomical axis for control of energy balance. Front Neuroendocrinol. 2002;23(1):2-40.

9. Skibicka KP, Grill HJ. Hindbrain leptin stimulation induces anorexia and hyperthermia mediated by hindbrain melanocortin receptors. Endocrinology. 2009;150(4):1705-1711.

10. Hayes MR, et al. Endogenous leptin signaling in the caudal nucleus tractus solitarius and area postrema is required for energy balance regulation. Cell Metab. 2010;11(1):77-83.

11. Williams DL, Baskin DG, Schwartz MW. Hindbrain leptin receptor stimulation enhances the anorexic response to cholecystokinin. Am J Physiol Regul Integr 
Comp Physiol. 2009;297(5):R1238-R1246.

12. Huo L, Maeng L, Bjorbaek C, Grill HJ. Leptin and the control of food intake: neurons in the nucleus of the solitary tract are activated by both gastric distension and leptin. Endocrinology. 2007;148(5):2189-2197.

13. Morton GJ, et al. Leptin action in the forebrain regulates the hindbrain response to satiety signals. J Clin Invest. 2005;115(3):703-710.

14. Ellacott KL, Halatchev IG, Cone RD. Characterization of leptin-responsive neurons in the caudal brainstem. Endocrinology. 2006;147(7):3190-3195.

15. Huo L, Grill HJ, Bjorbaek C. Divergent regulation of proopiomelanocortin neurons by leptin in the nucleus of the solitary tract and in the arcuate hypothalamic nucleus. Diabetes. 2006;55(3):567-573.

16. Mercer JG, Moar KM, Findlay PA, Hoggard N, Adam CL. Association of leptin receptor (OB-Rb), NPY and GLP-1 gene expression in the ovine and murine brainstem. Regul Pept. 1998;75-76:271-278.

17. Goldstone AP, et al. Leptin interacts with glucagon-like peptide-1 neurons to reduce food intake and body weight in rodents. FEBS Lett. 1997; 415(2):134-138.

18. Huo L, Gamber KM, Grill HJ, Bjorbaek C. Divergent leptin signaling in proglucagon neurons of the nucleus of the solitary tract in mice and rats.
Endocrinology. 2008;149(2):492-497.

19. Myers MG Jr, Leibel RL, Seeley RJ, Schwartz MW. Obesity and leptin resistance: distinguishing cause from effect. Trends Endocrinol Metab. 2010;21(11):643-651.

20. Munzberg H, Flier JS, Bjorbaek C. Region-specific leptin resistance within the hypothalamus of diet-induced obese mice. Endocrinology. 2004; 145(11):4880-4889

21. Ring LE, Zeltser LM. Disruption of hypothalamic leptin signaling in mice leads to early-onset obesity, but physiological adaptations in mature animals stabilize adiposity levels. J Clin Invest. 2010; 120(8):2931-2941.

\title{
Chronic lung allograft rejection and airway microvasculature: Is HIF-1 the missing link?
}

\author{
David S. Wilkes
}

Center for Immunobiology, Departments of Medicine, Microbiology and Immunology, Indiana University School of Medicine, Indianapolis, Indiana, USA.

\begin{abstract}
Chronic lung allograft rejection, known as obliterative bronchiolitis (OB), is the leading cause of death in lung transplant patients. Although $O B$ pathogenesis is not fully understood, in this issue of the JCI, Jiang and colleagues report that tissue hypoxia resulting in dysfunctional airway microvasculature precedes the airway fibrosis characteristic of $O B$. In addition, a relative deficiency of allograft endothelial cell-derived HIF- $1 \alpha$ contributes to this process. Data showing that overexpressing HIF-1 $\alpha$ restores the microvascular airway normoxia and prevents airway fibrosis highlight a novel role for vascular biology in $\mathrm{OB}$ pathogenesis.
\end{abstract}

Lung transplantation is the definitive therapy for many end-stage pulmonary diseases. However, the long-term survival of the lung transplant recipient is limited by chronic rejection known as obliterative bronchiolitis (OB, aka bronchiolitis obliterans syndrome [BOS]) (1). In fact, OB largely accounts for the 50\% five-year survival rate after lung transplantation, which is the worst of all solid organ allografts (1). The mechanisms leading to $\mathrm{OB}$ continue to be an area of intensive investigation, and studies have revealed that immune responses to the donor (alloimmunity) as well as exposure of autoantigens may participate in the rejection response (reviewed in ref. 2). The convergent result of these injurious activities is the development of a fibroproliferative process resulting in small airway occlusion, which is the pathologic hallmark of OB.

Conflict of interest: David S. Wilkes is the co-founder of ImmuneWorks Inc., which is developing novel forms of treatments for immunologically mediated lung diseases. The author has also received income as a consultant for ImmuneWorks Inc.

Citation for this article: J Clin Invest. 2011; 121(6):2155-2157. doi:10.1172/JCI58329.

\section{OB stems from hypoxia}

Implicit in the term chronic rejection is the idea that immune mechanisms are likely to predominate in $\mathrm{OB}$. However, even prior to immune activation, the lung may be at risk for OB due to airway hypoxia resulting from transplant-related microvasculature interruption. The lung has three vascular supplies, the pulmonary arteries, the pulmonary veins, and the bronchial artery, and is the only solid organ for which allograft does not involve direct systemic arterial reconnection at the time of surgery. The pulmonary arterial/venous circulation is restored in the transplanted lung. However, the bronchial artery, the only source of fully oxygenated blood under systemic arterial pressure, is not reanastomosed after transplantation due to the significant technical complexities associated with this procedure.

The lack of an intact bronchial artery circulation leads to impaired microcirculation, suggesting that prolonged airway hypoxia contributes to $O B$. In fact, previous studies from the Nicolls group have confirmed that airway epithelial hypoxia occurs following clinical lung transplantation (3), and other researchers have reported that the loss of the microvasculature in small airways precedes OB $(4,5)$. Hypoxia, a key adverse effect of losing the vascular supply, may induce profound changes in airway epithelium. One of these effects could be the induction of epithelial mesenchymal transition (EMT), a process implicated in fibrogenesis in many organs, including the lung (6). Indeed, studies from the laboratory of Jacob Sznajder demonstrated that both moderate and severe hypoxia induced EMT (6). These findings have direct relevance to lung transplantation, since recent studies have detected EMT in OB lesions (7-9).

Recent studies strongly suggest that hypoxia may lower the threshold to induce adaptive immune responses known to have key roles in acute lung transplant rejection. Due to the presence of bronchus-associated lymphoid tissue, interstitial and interepithelial dendritic cells, a full complement of lymphocytes, and macrophages, the lung is uniquely able to mount adaptive immune responses in the absence of any secondary lymphoid organs $(10,11)$. Indeed, in essence, the lung is a lymph node with alveoli (2). What is the relationship of immunity to chronic hypoxia and rejection in the transplanted lung? Recent studies indicate that hypoxia may augment immune activation (12) and that alloimmune activation occurs within the transplanted lung (10). For example, hypoxia induces the activation of dendritic cells that stimulate alloimmunity, produce proinflammatory cytokines, and activate Th17 cells that produce IL-17 $(13,14)$. In addition, production of IL-17 